Abdel-Malek, K. and Yeh, H.J., (1997), "Path Trajectory Verification for Robot Manipulators in a Manufacturing Environment" IMechE Journal of Engineering Manufacture, Vol. 211, part B, pp. 547-556.

\title{
Path Trajectory Verification for Robot Manipulators in a Manufacturing Environment
}

\author{
K. Abdel-Malek ${ }^{1}$, B.Sc., M.Sc., Ph.D. \\ H. J. Yeh, B.Sc., M.Sc., Ph.D.
}

Department of Mechanical Engineering and Center for Computer-Aided Design, The University of Iowa, Iowa City, IA 52242

In a manufacturing environment where a robotic arm is programmed to follow a specified trajectory such as in welding, painting, and soldering, it is often the case that the arm reaches a singular configuration, where the program is stopped, the arm is switched to a new configuration, and the motion is continued. This difficulty has been a long standing problem. This paper presents a mathematical formulation for verifying whether a trajectory can be completed, uninterrupted, avoiding halting of a planned path. In some cases, this formulation also allows the selection of an initial configuration to ensure a smooth path trajectory. The paper presents an analytical formulation for determining barriers to motion inside the workspace of manipulator arms. Crossability analysis of the end-effector on a barrier are addressed. A criterion for selecting an initial configuration that would result in an un-interrupted motion is introduced. The mathematical theory is validated through numerical examples of planar and spatial manipulator arms.

Keywords: Trajectory planning, robot singularities, trajectory verification, robot path, and manipulator kinematics

NOTATION

$\mathbf{x}$ Position vector

$\Phi$ Constraint vector function

q Vector of generalized coordinates

${ }^{i} \mathbf{T}_{j} \quad(4 \times 4) \quad$ transformation matrix relating body $i$ to $j$

${ }^{i} \mathbf{R}_{j}(3 \times 3)$ rotation matrix between frames $i$ and $j$

$a_{i}$ midpoint of an interval $b_{i} \quad$ half range of an interval

$\Phi_{\mathbf{q}}$ Jacobian of $\Phi$ with respect to $\mathbf{q}$

$S \quad$ A set

$\Re^{n} \quad$ Space of $\mathrm{n}$-generalized coordinates

u A vector of generalized coordinates

$\mathrm{p}_{i} \quad \mathrm{~A}$ singular set of constant generalized coordinates

$\zeta_{o} \quad$ A normal vector

$\Psi \quad$ A subsurface parametrized same as its corresponding $\Phi$ but bounded

\footnotetext{
${ }^{1}$ Corresponding author
} 


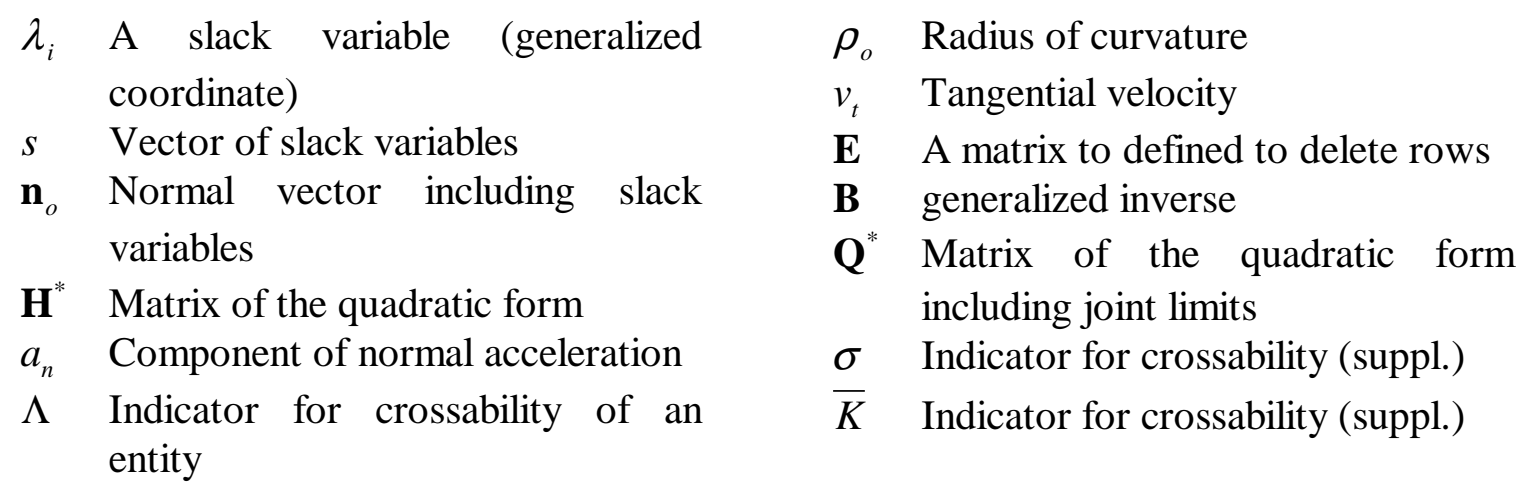

\section{INTRODUCTION}

Many tasks performed by a manipulator arm in a manufacturing environment such as arc welding, spray painting, continuous soldering, and deburring, require that the end-effector follows a prescribed trajectory as depicted in Fig. 1. During this process of motion from point A to B, singular behavior of the manipulator may occur. This type of problem has been well documented (1). Many researchers have referred to this problem as determining the admissibility of paths in the task space (2), in joint space (3), or trajectory verification in the workspace (4). Although this type of problem has received considerable attention in recent years, a general formulation for determining whether the specified trajectory can be followed without interruption remains an open problem. Two search methods to determine the path that satisfies all constraints were presented by Milton and Jennings (5). Other works that have dealt with the subject are Althofer et al. (6), Seereeram and Wen (7), Agrawal and $\mathrm{Xu}(\mathbf{8})$, and Nenchev (9).

Studies that have addressed difficulties in the control of manipulators due to the appearance of interior curves and surfaces were reported by Waldron (10) and Nielsen et al. (11). In the latter work, the controllability of a mechanical arm is discussed from a differential geometry point of view. The fundamental concept of crossable and noncrossable surfaces inside a manipulator's workspace was addressed by Oblak and Kohli (12). Their report introduced the concept of a crossable surface but did not establish generalized criteria.

Recently, Haug et al. (13) presented a numerical algorithm for identifying and analyzing barriers to output control of manipulators using first and second-order Taylor approximations of the output in selected directions. More recently, Abdel-Malek and Yeh (14) presented an analytical method by which all singular surfaces and curves can be analytically determined for a serial manipulator with joint limits. The work was extended and compared with numerical techniques in (15). The work is extended in this paper to the verification of a trajectory subject to the existence of singular geometric entities inside the workspace.

In many situations, however, there are many choices for selecting an initial configuration for the manipulator at point A (Fig. 1). The choice of initial configuration is critical to the completion of the trajectory without interruption. During the motion from point A to B, 
the manipulator may reach a configuration at which one or more joints are at their limits. At this configuration, the motion halts, and a different configuration is specified. To illustrate, consider the manipulator arm shown in Fig. 2, where it is required that a point on the end-effector follow the trajectory from A to B. As the end-effector is moving from point $\mathrm{A}$ to point $\mathrm{B}$ (the orientation is not considered) the manipulator reaches a configuration at point $\mathrm{Q}$ where it is no longer possible to continue the motion because joints 2 and 3 reached their limits. At this point, another configuration is specified (as shown in Fig. 3) and the motion continues. Therefore, although the motion can be completed, an interruption has occurred that may cause damage to the manufacturing process.

A choice of a different initial configuration at point A could have resulted in a smooth motion of the trajectory without interruption, where the end-effector is able to move from point A to point B without encountering singular behavior.

It is evident that the formulation for selecting an appropriate trajectory should take into consideration the joint limits of the manipulator. In this paper, a mathematical formulation for determining curves and surfaces inside the workspace upon which the manipulator may exhibit singular behavior will be introduced. For a general multi degree-of-freedom serial manipulator, it will be shown that there are surfaces inside the workspace for which the end-effector cannot cross, or may cross in one direction only. Those surfaces represent certain configurations of the manipulator for which joint constraints are active or for which the manipulator has assumed a singular configuration such as the case that may occur when two consecutive joints are parallel. The direction of movement on those surfaces will also be discussed. Verification of the trajectory is then performed by intersecting the path curve with internal barriers. Selecting an initial configuration at point A will be detrimental to the successful completion of the planned trajectory without interruption. The method will be presented through a simple example of the planar three degree-of-freedom manipulator (Fig. 1). A general formulation will then be deduced and a complete numerical example of a spatial manipulator arm will be presented.

\section{SINGULAR GEOMETRIC ENTITIES INSIDE THE WORKSPACE}

Inside the workspace of a mechanical manipulator, there exist curves and surfaces that represent impediments to motion that may cause output control difficulties $(\mathbf{1 3}, \mathbf{1 5})$. The objective of this section is to determine these barriers.

The position vector of a point on the end-effector of a serial manipulator can be written in terms of joint coordinates as

$$
\mathbf{x}=\Phi(\mathbf{q})
$$

where $\mathbf{q} \in \mathfrak{R}^{n}$ is the vector of $n$-generalized coordinates, and $\Phi(\mathbf{q})$ can be obtained from the multiplication of the homogeneous transformation matrices defined by the $\mathrm{D}-\mathrm{H}$ representation method $(\mathbf{1 6}, \mathbf{1 7})$ as

$$
{ }^{0} \mathbf{T}_{n}={ }^{0} \mathbf{T}_{1}{ }^{1} \mathbf{T}_{2} \cdots{ }^{n-1} \mathbf{T}_{n}=\left[\begin{array}{cc}
{ }^{i} \mathbf{R}_{j}(\mathbf{q}) & \Phi(\mathbf{q}) \\
\mathbf{0} & 1
\end{array}\right]
$$


where ${ }^{i} \mathbf{R}_{j}$ is the rotation matrix and ${ }^{i} \mathbf{T}_{j}$ is the homogeneous transformation matrix relating bodies $i$ to $j$. In order to extend the formulation to include joint limits in the form of inequality constraints such as $q_{i}^{\min } \leq q_{i} \leq q_{i}^{\max }$, a transformation is introduced as

$$
q_{i}=a_{i}+b_{i} \sin \lambda_{i}
$$

where $a_{i}=\left(q_{i}{ }^{\max }+q_{i}{ }^{\min }\right) / 2$ and $b_{i}=\left(q_{i}{ }^{\max }-q_{i}{ }^{\min }\right) / 2$ are the mid point and half range of the inequality constraint and $\lambda$ is a slack variable. The position constraint function is then written in terms of the extended vector $\mathbf{s}=\left[\begin{array}{lll}\lambda_{1} & \lambda_{2} & \ldots \lambda_{n}\end{array}\right]^{T}$ such that

$$
\mathbf{x}=\Phi^{*}(\mathbf{s})=\left[\Phi\left(\mathbf{q}\left(\lambda_{i}\right)\right)\right]
$$

End-effector velocities can be determined by deriving equation (4) with respect to time and using the chain rule as

$$
\dot{\mathbf{x}}=\Phi_{\mathbf{q}} \mathbf{q}_{\mathbf{s}} \dot{\mathbf{s}}
$$

where $\mathbf{q}_{\mathbf{s}}=\partial \mathbf{q} / \partial \lambda_{j}$ and $\dot{\mathbf{s}}=d \mathbf{s} / d t$ and $\Phi_{\mathbf{q}}$ is the Jacobian defined by

$$
\Phi_{\mathbf{q}}=\left[\partial \Phi_{i}(\mathbf{q}) / \partial q_{j}\right]
$$

Define the subvector of $\mathbf{q}$ as a set of constant generalized coordinates $\mathrm{p}_{i} \in \Re^{m}$ where $m \leq n-1$, and $\mathbf{q}=\mathbf{u} \cup \mathrm{p}_{i}$. Singular sets $\mathrm{p}_{i}$ of the manipulator can be obtained from studying the dimension of the null space of $\Phi_{\mathbf{q}}^{T}(\mathbf{q})$, defined by Abdel-Malek and Yeh (14) as the set

$$
\mathbf{S}=\left\{\mathrm{p}_{i} \in \Re^{m} ; \operatorname{dim}\left[\operatorname{Null}\left(\Phi_{\mathbf{q}}^{T}(\mathbf{q})\right)\right] \geq 1, \mathbf{q}=\left[\begin{array}{ll}
\mathbf{u} & \mathbf{p}_{i}
\end{array}\right] \text { for some constant } \mathbf{p}_{i}\right\}
$$

where $\mathbf{u} \in \mathfrak{R}^{n-m}$ is the vector of generalized coordinates that are not in $\mathrm{p}_{i}$. On a singular surface, the term $\left.\Phi_{\mathbf{q}} \mathbf{q}_{\mathbf{s}}\right|_{\mathbf{q}_{o}, s_{o}}$ is rank-deficient. Therefore, the rows of $\Phi_{\mathbf{q}} \mathbf{q}_{\mathbf{s}}$ are dependent and there exists a set of constants $\mathbf{n}_{o}=\left[\begin{array}{lll}n_{1} & n_{2} & n_{3}\end{array}\right]^{T}$, that satisfy

$$
\left[\Phi_{\mathbf{q}} \mathbf{q}_{\mathbf{s}}\right] \mathbf{n}_{o}^{T}=\mathbf{0}
$$

where $\mathbf{n}_{o}$ is the basis to the null space of $\left[\Phi_{\mathbf{q}} \mathbf{q}_{\mathbf{s}}\right]$.

It was shown that substituting $\mathrm{p}_{i}$ into $\Phi(\mathbf{q})$ yields singular surfaces and curves parametrized by $\Psi(\mathbf{u})$ where $\mathbf{u}$ is the new vector of generalized coordinates such that

$$
\Phi\left(\mathbf{u}, \mathrm{p}_{i}\right)=\Psi(\mathbf{u})
$$

and $\mathbf{n}_{o}$ is indeed the normal vector to these geometric entities defined by equation (9).

To demonstrate the above formulation, consider the planar 3DOF manipulator shown in Fig. 1. The position vector of a point on the end-effector of the planar three degree-offreedom manipulator is defined by

$$
\Phi(\mathbf{q})=\left[\begin{array}{c}
3 \cos q_{1}+2 \cos \left(q_{1}+q_{2}\right)+\cos \left(q_{1}+q_{2}+q_{3}\right) \\
3 \sin q_{1}+2 \sin \left(q_{1}+q_{2}\right)+\sin \left(q_{1}+q_{2}+q_{3}\right)
\end{array}\right]
$$

where $\mathbf{q}=\left[\begin{array}{lll}q_{1} & q_{2} & q_{3}\end{array}\right]^{T}$ is the vector of generalized coordinates and the joints are subject to the following inequality constraints $-\frac{\pi}{3} \leq q_{i} \leq \frac{\pi}{3}$. In order to determine the 
manipulator's singular characteristics, it is necessary to study the manipulator's $(2 \times 3)$ Jacobian $\Phi_{\mathbf{q}}=\left[\partial \Phi_{i} / \partial q_{j}\right]$ where

$\Phi_{\mathbf{q}}=\left[\begin{array}{ccc}-3 \sin q_{1}-2 \sin \left(q_{1}+q_{2}\right)-\sin \left(q_{1}+q_{2}+q_{3}\right) & -2 \sin \left(q_{1}+q_{2}\right)-\sin \left(q_{1}+q_{2}+q_{3}\right) & -\sin \left(q_{1}+q_{2}+q_{3}\right) \\ 3 \cos q_{1}+2 \cos \left(q_{1}+q_{2}\right)+\cos \left(q_{1}+q_{2}+q_{3}\right) & 2 \cos \left(q_{1}+q_{2}\right)+\cos \left(q_{1}+q_{2}+q_{3}\right) & \cos \left(q_{1}+q_{2}+q_{3}\right)\end{array}\right]$

The dimension of the nullspace of the Jacobian is studied. In this case, only one singular set results from the $\operatorname{dim}\left[\operatorname{Null}\left(\Phi_{\mathbf{q}}^{T}(\mathbf{q})\right)\right] \geq 1$ defined by $S_{1}: \mathrm{p}_{1}=\left\{q_{2}=0, q_{3}=0\right\}$.

Singularities resulting from the reduced order manipulator are $S_{2}=\left\{\mathbf{p}_{i}, i=2 \ldots 7\right\}$ where

$\mathrm{p}_{2}=\left\{q_{1}=-\pi / 3, q_{3}=0\right\}, \mathrm{p}_{3}=\left\{q_{1}=\pi / 3, q_{3}=0\right\}, \mathrm{p}_{4}=\left\{q_{1}=-\pi / 3, q_{3}=\tan ^{-1}(\sqrt{3} / 2)\right\}$,

$\mathrm{p}_{5}=\left\{q_{1}=\pi / 3, q_{3}=-\tan ^{-1}(\sqrt{3} / 2)\right\}, \mathrm{p}_{6}=\left\{q_{1}=-\pi / 3, q_{2}=\tan ^{-1}(\sqrt{3} / 5)\right\}$, and

$\mathrm{p}_{7}=\left\{q_{1}=\pi / 3, q_{2}=-\tan ^{-1}(\sqrt{3} / 5)\right\}$. Singularity sets resulting from a combination of joint limits are $\left\{\mathrm{p}_{i}=\left\{q_{i}^{o}, q_{j}^{o}\right\}, i \neq j, i, j=8 . . .19\right\}$ where $q_{i}^{o}$ and $q_{j}^{o}$ are the limits of the inequality constraints. Substituting each singularity set $\mathrm{p}_{i},\{i=1 \ldots 19\}$ into equation (10) yields singular curves $\Psi_{i}$ part of which are shown in Fig. (4).

\section{IMPEDIMENTS TO MOTION}

The determination of the crossability of a surface/curve inside the workspace can be established from the definiteness properties of a quadratic form representing the normal acceleration of the system. The acceleration of the end-effector can be written in matrix form as

$$
\ddot{\mathbf{x}}=\dot{\mathbf{s}}\left\{\mathbf{q}_{\mathbf{s}}^{T} \Phi_{\mathbf{q q}} \mathbf{q}_{\mathbf{s}}+\sum_{i=1}^{n} \frac{d \Phi}{d q_{i}} \cdot\left[q_{i}\right]_{\mathrm{ss}}\right\} \dot{\mathbf{s}}+\left[\Phi_{\mathbf{q}} \mathbf{q}_{\mathbf{s}}\right] \ddot{\mathbf{s}}
$$

where $\Phi_{\mathbf{q q}}=\left[\partial^{2} \Phi / \partial q_{i} \partial q_{j}\right]$ and $\left[q_{i}\right]_{\mathrm{ss}}=\left[\partial^{2} q_{i} / \partial s_{i} \partial s_{j}\right]$. To obtain the component of normal acceleration, $\ddot{\mathbf{x}}$ is projected onto the normal $\mathbf{n}_{o}$. Multiplying both sides of equation (12) by the vector $\mathbf{n}_{o}^{T}$ eliminates the last term of the right hand side (definition of the normal as the basis of the null space in equation 8). The component of the normal acceleration is then

where

$$
a_{n}=\mathbf{n}_{o}^{T} \ddot{\mathbf{x}}=\dot{\mathbf{s}}^{T} \mathbf{H}^{*} \dot{\mathbf{S}}
$$

$$
\mathbf{H}^{*}\left(\mathbf{q}_{o}, \mathbf{s}_{o}\right)=\mathbf{q}_{\mathbf{s}}^{T}\left[\mathbf{n}_{o}^{T} \Phi\right]_{\mathbf{q q}} \mathbf{q}_{\mathbf{s}}+\sum_{i=1}^{n} \frac{d\left(\mathbf{n}_{o}^{T} \Phi\right)}{d q_{i}} \cdot\left[q_{i}\right]_{\mathrm{ss}}
$$

In order to determine whether the manipulator's end-effector can cross a singular entity, it is necessary to study acceleration components that would cause motion normal to the surface or curve. However, to generalize the discussion, the normal curvature of a singular surface is considered. The criteria for the end-effector to cross a singular surface is specified by the normal acceleration indicator $\Lambda$ given by

$$
\Lambda=a_{n}-\frac{\left|v_{t}^{2}\right|}{\rho_{o}}
$$


where $v_{t}=\dot{\mathbf{x}}$ is the tangential velocity, $1 / \rho_{o}$ is the normal curvature of the singular surface with respect to the tangent direction of $v_{t}$ ( $\rho_{o}$ is the radius of curvature), and the component $\left|v_{t}^{2}\right| / \rho_{o}$ defined in terms of the parametrized entity equation is

$$
\left|v_{t}^{2}\right| / \rho_{o}=\dot{\mathbf{u}}^{T}\left[\mathbf{n}^{T} \Psi\right]_{\mathbf{u}} \dot{\mathbf{u}}
$$

If $a_{n}$ and $v_{t}$ are on the same side of the surface, then the end-effector admits motion in the normal direction of this side of the surface (Fig. 5a). In specific, a positive value of $\Lambda$ indicates that the end-effector will move in the normal direction away from the center, a zero value indicates the manipulator moving on the surface, and a negative value indicates the manipulator admitting motion towards the center of curvature as illustrated in Fig. 5b.

At a singular configuration, the tangent velocity in terms of the entity $\Psi$ or in terms of the constant constraint function $\Phi$, can be written as

$$
\Psi_{\mathbf{u}} \dot{\mathbf{u}}=\Phi_{\mathrm{q}} \dot{\mathbf{q}}
$$

In order to obtain an expression for $\dot{\mathbf{u}}$, an inverse for $\Psi_{\mathbf{u}}$ is needed. Because $\Psi_{\mathbf{u}}$ is a nonsquare $(3 \times 2)$ matrix, define a matrix $\mathbf{E}$ such that

$$
\begin{aligned}
& \mathbf{E}=\left[\begin{array}{lll}
1 & 0 & 0 \\
0 & 1 & 0
\end{array}\right] \text { if the first and second rows of } \Psi_{\mathrm{u}} \text { are independent } \\
& \mathbf{E}=\left[\begin{array}{lll}
1 & 0 & 0 \\
0 & 0 & 1
\end{array}\right] \text { if the first and third rows of } \Psi_{\mathrm{u}} \text { are independent } \\
& \mathbf{E}=\left[\begin{array}{lll}
0 & 1 & 0 \\
0 & 0 & 1
\end{array}\right] \text { if the second and third rows of } \Psi_{\mathrm{u}} \text { are independent }
\end{aligned}
$$

Multiply both sides of equation (17) from the left by $\mathbf{E}$ yields

$$
\mathbf{E} \Psi_{\mathbf{u}} \dot{\mathbf{u}}=\mathbf{E} \Phi_{\mathbf{q}} \dot{\mathbf{q}}
$$

Multiplying both sides of equation (21) by the inverse of $\left[\mathbf{E} \Psi_{\mathbf{u}}\right]$ yields

$$
\dot{\mathbf{u}}=\left[\mathbf{E} \Psi_{\mathbf{u}}\right]^{-1} \mathbf{E} \Phi_{\mathbf{q}} \mathbf{q}_{\mathbf{s}} \dot{\mathbf{s}}
$$

where the chain rule $\dot{\mathbf{q}}=\mathbf{q}_{\mathbf{s}} \dot{\mathbf{s}}$ is used. Equation (22) can be written as

$$
\dot{\mathbf{u}}=\mathbf{B} \Phi_{\mathbf{q}} \mathbf{q}_{\mathbf{s}} \dot{\mathbf{s}}
$$

where the matrix $\mathbf{B}$ is a generalized inverse of $\Psi_{\mathrm{u}}$ defined by

$$
\mathbf{B} \equiv\left[\mathbf{E} \Psi_{\mathbf{u}}\right]^{-1} \mathbf{E}
$$

In this extended formulation (considering joint limits), the quadratic form of the normal acceleration indicator $\Lambda$ is written in terms of the $\mathbf{Q}^{*}$ matrix as

$$
\Lambda=a_{n}-\frac{\left|v_{t}\right|^{2}}{\rho_{o}}=\dot{\mathbf{s}}^{T} \mathbf{Q}^{*} \dot{\mathbf{s}}
$$

where

$$
\mathbf{Q}^{*}=\mathbf{H}^{*}-\mathbf{q}_{\mathbf{s}}^{T} \Phi_{\mathbf{q}}^{T} \mathbf{B}^{T}\left[\mathbf{n}^{T} \Psi\right]_{\mathbf{u u}} \mathbf{B} \Phi_{\mathbf{q}} \mathbf{q}_{\mathbf{s}}
$$


Definiteness properties of the quadratic form of equation (26) define the crossability of a singular surface/curve. If $\mathbf{Q}^{*}$ is indefinite (has both positive and negative eigenvalues), the singular surface/curve is crossable. Because of the transformation used to include joint limits in this formulation, the derivative of equation (3) at a joint limit yields a zero velocity. The nullification of joint velocities gives rise to an apparent loss of information from the matrix of the quadratic form (equation 26). Therefore, these supplemental criteria are introduced. If $\mathbf{Q}^{*}$ is either positive semi-definite or negative semi-definite, the following additional criteria must be evaluated.

(a) If $\mathbf{n}_{o}$ is not perpendicular to $\mathbf{x}_{q_{i}}$, then define $\sigma$ as a projection of a variational motion $\mathbf{x}_{q_{i}} \delta q_{i}$ onto the normal, evaluated by

$$
\sigma=\mathbf{n}^{T} \mathbf{x}_{q_{i}} \delta q_{i}
$$

where $\delta q_{i}$ is given a magnitude of \pm 1 as follows

$$
\delta q_{i}=\left\{\begin{array}{lll}
+1 & \text { if } q_{i} \text { is at lower bound } \\
-1 & \text { if } q_{i} \text { is at upper bound }
\end{array}\right.
$$

(b) If $\mathbf{n}_{o}$ is perpendicular to $\mathbf{x}_{q_{i}}$, then the difference in the normal curvature between the two surfaces as

$$
\bar{K}=\mathbf{x}_{q_{i}}^{T} \mathbf{B}^{T}\left[\mathbf{n}^{T} \mathbf{x}\right]_{\mathrm{uu}} \mathbf{B} \mathbf{x}_{q_{i}}-\left[\mathbf{n}^{T} \mathbf{x}\right]_{q_{i} q_{i}}
$$

where equation (29) is the difference in curvature between the surfaces.

(i) If any of $\sigma$ or $\bar{K}$ has a different sign than the nonzero eigenvalues of $\mathbf{Q}^{*}$, the singular surface/curve is crossable.

(ii) If $\sigma$ and $\bar{K}$ have the same sign as the nonzero eigenvalues of $\mathbf{Q}^{*}$, the singular surface/curve is non-crossable.

The direction along which the end-effector can move, either in the positive or negative direction of $\mathbf{n}$, will be in the same sense of signs of $\sigma$ and $\bar{K}$.

The above criteria establish whether a manipulator at a singular surface may exhibit output control difficulties, i.e., a manipulator following a trajectory, crossing one of those barriers will have to halt.

\section{VERIFYING A TRAJECTORY}

For a trajectory defined as a curve in space parametrized in terms of one variable $\mathbf{x}^{t r}(t)$, it is necessary to determine all intersections of this curve with non-crossable singular entities $\Psi(\mathbf{u})$ inside the workspace. Therefore, at each intersection point satisfying

$$
\mathbf{x}^{t r}(t)-\Psi(\mathbf{u})=\mathbf{0}
$$

it is necessary to study the crossability criteria characterized by the definiteness properties of the matrix $\mathbf{Q}^{*}$.

For the planar three-bar example, it is first necessary to evaluate the criteria above for each independent segment, i.e., curves that cross other curves are segmented. For 
example, consider a point $\mathbf{p}_{1}$ on curve $\Psi_{19}$ at $q_{1}=\pi / 4, q_{2}=\pi / 3$, and $q_{3}=\pi / 3$. Evaluating the normal $\mathbf{n}$ from the basis of the null space of

$$
\left[\Phi_{\mathbf{q}} \mathbf{q}_{\lambda}\right]^{T}=\left[\begin{array}{ccc}
-3.4765 & 0 & 0 \\
0.9315 & 0 & 0
\end{array}\right]
$$

at $\mathbf{p}_{1}$ yields the normal $\mathbf{n}=\left[\begin{array}{ll}0.2588 & 0.9659\end{array}\right]^{T}$. Evaluating the matrix $\mathbf{H}^{*}$ of equation (14) yields

$$
\mathbf{H}^{*}=\left[\begin{array}{ccc}
-2.4930 & 0 & 0 \\
0 & 2.0944 & 0 \\
0 & 0 & 1.0472
\end{array}\right]
$$

Since this is a planar example, the singular curve is parametrized by only one joint variable (i.e., $\Psi_{19}\left(q_{1}\right)$, therefore, $\mathbf{x}_{q_{1}}$ is a $(2 \times 1)$ matrix. The matrix $\mathbf{E}$ is therefore a $\mathbf{E}=\left[\begin{array}{ll}1 & 0\end{array}\right]$ vector and $\mathbf{B}=\left[\mathbf{E} \Psi_{\mathbf{u}}\right]^{-1} \mathbf{E}=\left[\begin{array}{ll}-0.1992 & 0\end{array}\right]$. The matrix $\mathbf{Q}^{*}$ of the quadratic form is evaluated as

$$
\mathbf{Q}^{*}=\left[\begin{array}{ccc}
0 & 0 & 0 \\
0 & 2.094 & 0 \\
0 & 0 & 1.047
\end{array}\right]
$$

The eigenvalues of $\mathbf{Q}^{*}$ are evaluated $\{0,1.047$, and 2.094$\}$, which indicates a positive semi-definite quadratic form. Since the constraints of joints 2 and 3 are active (upper bounds) and $\mathbf{x}_{q_{2}}$ and $\mathbf{x}_{q_{3}}$ are not parallel to $\mathbf{n}$, the additional value of $\sigma$ is evaluated. For $q_{2}$ at its upper limit, $\delta q_{2}=-1$, and $\sigma=\mathbf{n}^{T} \mathbf{x}_{q_{2}} \delta q_{2}=2$ which is positive. For $q_{3}$, choose $\delta q_{3}=-1$ and evaluate $\sigma=\mathbf{n}^{T} \mathbf{x}_{q_{3}} \delta q_{3}=1$ which is also positive. Both values for $\sigma$ are positive (same signs as the eigenvalues of $\mathbf{Q}^{*}$ ). Therefore, surface $\Psi_{19}$ only admits movement into the positive direction of the normal $\mathbf{n}$ (i.e., only towards the internal of the workspace) as expected.

To further demonstrate, consider point $\mathbf{p}_{2}$ on $\Psi_{6}$ at $q_{1}=\pi / 8, q_{2}=\tan ^{-1}(\sqrt{3} / 5)$, and $q_{3}=-\pi / 3$. The matrix $\mathbf{Q}^{*}$ of the quadratic form is indefinite (both negative and positive eigenvalues) indicating a crossable curve at $\mathbf{p}_{2}$. Similar analysis is performed for all curve segments. For example, the curve $\Psi_{8}$ is crossable for the segment of $0.7137 \leq q_{3} \leq \pi / 3$; non-crossable for the segment $0 \leq q_{3} \leq 0.7137$; and crossable for $-\pi / 3 \leq q_{3} \leq 0$. The curve $\Psi_{9}$ is non-crossable for the segment of $0 \leq q_{3} \leq \pi / 3$; crossable for the segment $-0.7137 \leq q_{3} \leq 0$; and non-crossable for $-\pi / 3 \leq q_{3} \leq-0.7137$. All barriers are numbered and shown in Fig. (6a). Only crossable curves are shown in Fig. (6b) while non-crossable lines are removed. The direction of admissible movement is also shown by arrows on each curve segment in (6b).

Consider the trajectory shown in Fig. 1 and reproduced in Fig. 7. It is required to study this trajectory such that the end-effector can travel from point A to B without interruption. 
The curve is intersected with only those singular entities that are non-crossable (barriers) since they may be impediments to motion. Performing the intersection defined by equation (30) yields point $\mathrm{Q}$ on singular curve $\Psi_{18}$ where $\mathrm{p}_{18}=\left\{q_{2}=\pi / 3, q_{3}=-\pi / 3\right\}$.

In order to plan a trajectory that would not be interrupted requires knowledge of all inverse kinematic solutions along the path. The trajectory planner generates a number of solutions, one of which is selected. The initial configuration is chosen such that when the end-effector reaches point $\mathrm{Q}$, it is not in a singular configuration.

To illustrate, Fig. 8 depicts the trajectory planned for the three-bar manipulator in the workspace. An alternative configuration is specified at point $\mathrm{Q}$ as shown in Fig. 8. Let the manipulator start with the initial configuration specified by posture III, such that at point $\mathrm{Q}$, the manipulator is in posture II, therefore, sustaining a smooth motion and avoiding the configuration defined by posture I.

\section{A SPATIAL EXAMPLE}

In general, manipulators used in manufacturing are spatial with three or more degrees-offreedom. The singular entities generated inside the workspace of such manipulators are surfaces parametrized in terms of two or three parameters. Consider, for example, the five degree-of-freedom manipulator shown in Fig. (9). The joints are constrained as $0 \leq q_{1} \leq 2 \pi,-\pi / 4 \leq q_{2} \leq 5 \pi / 4,0 \leq q_{3} \leq 5,0 \leq q_{4} \leq 2 \pi$, and $-\pi / 2 \leq q_{5} \leq \pi / 2$.

The position vector of point $\mathbf{p}$ in Fig. 9 is determined as

$$
\Phi(\mathbf{q})=\left[\begin{array}{c}
-5 \sin q_{5} \cos q_{1} \sin q_{2} \sin q_{4}-5 \sin q_{5} \sin q_{1} \cos q_{4}+5 \cos q_{1} \cos q_{2} \cos q_{5}+15 \cos q_{1} \cos q_{2}+q_{3} \cos q_{1} \cos q_{2} \\
-5 \sin q_{5} \sin q_{1} \sin q_{2} \sin q_{4}+5 \sin q_{5} \cos q_{1} \cos q_{4}+5 \sin q_{1} \cos q_{2} \cos q_{5}+15 \sin q_{1} \cos q_{2}+q_{3} \sin q_{1} \cos q_{2} \\
5 \cos q_{2} \sin q_{4} \sin q_{5}+5 \sin q_{2} \cos q_{5}+15 \sin q_{2}+q_{3} \sin q_{2}+25
\end{array}\right]
$$

Singularity analysis yields the following singular sets.

$\begin{array}{ll}\Psi_{1}: q_{2}=\pi / 2, q_{4}=0, \text { and } q_{5}=\pi / 2 & \Psi_{7}: q_{3}=5, q_{4}=0, \text { and } q_{5}=0 \\ \Psi_{2}: q_{2}=-\pi / 4, q_{4}=0, \text { and } q_{5}=0 & \Psi_{8}: q_{2}=\pi / 2, q_{3}=5 \text {, and } q_{4}=0 \\ \Psi_{3}: q_{2}=-\pi / 4, q_{4}=\pi / 2, \text { and } q_{5}=\pi / 2 & \Psi_{9}: q_{2}=-\pi / 4, q_{3}=0 \text {, and } q_{4}=\pi / 2 \\ \Psi_{4}: q_{2}=-\pi / 4, q_{4}=3 \pi / 2 \text {, and } q_{5}=\pi / 2 & \Psi_{10}: q_{2}=-\pi / 4, q_{3}=5 \text {, and } q_{4}=\pi / 2 \\ \Psi_{5}: q_{3}=0, q_{4}=0, \text { and } q_{5}=0 & \Psi_{11}: q_{3}=0 \text { and } q_{5}=\pi / 2 \\ \Psi_{6}: q_{2}=\pi / 2, q_{3}=0, \text { and } q_{4}=0 & \Psi_{12}: q_{3}=5 \text { and } q_{5}=\pi / 2\end{array}$


Substituting each singularity set into equation (31) yields a parametric equation of a singular surface. Note that singular surfaces $\Psi_{11}$ and $\Psi_{12}$ are two irregular parametric surfaces since they are parametrized in three variables $\left(q_{1}, q_{2}\right.$, and $\left.q_{4}\right)$. A cross section of the workspace of the 5-DOF manipulator depicting all singular surfaces is shown in Fig. (10). Because it is possible to obtain parametric equations of all singular entities, a mathematics symbolic manipulator is used to depict the entities.

Crossability of singular surfaces can now be determined. For example, consider a point located on surface $\Psi_{11}$ at $q_{1}=\pi / 2, q_{2}=\pi / 4, q_{3}=0, q_{4}=\pi / 2$, and $q_{5}=\pi / 2$. The normal vector is evaluated as $\mathbf{n}=\left[\begin{array}{lll}0 & 1 / \sqrt{5} & 2 / \sqrt{5}\end{array}\right]^{T}$ and the Eigenvalues of the $\mathbf{Q}^{*}$ matrix are $\{0,0,0,2.3717,7.4509\}$. Since the quadratic form of equation (25) is positive semi-definite ( $q_{3}$ and $q_{5}$ have active constraints) then equation (27) is used to evaluate $\sigma$. Both values of $\sigma$ for $q_{3}$ and $q_{5}$ are positive (0.9487 and 4.7434). Therefore, $\Psi_{11}$ is non-crossable (only in the positive direction of $\mathbf{n}$ ). Similarly for a point on surface $\Psi_{11}$ located at $\left(q_{1}=\pi / 2, q_{2}=\pi / 4, q_{3}=5, q_{4}=\pi / 2\right.$, and $\left.q_{5}=\pi / 2\right)$, the

normal vector is evaluated as $\mathbf{n}=\left[\begin{array}{lll}0 & 3 / \sqrt{34} & 5 / \sqrt{34}\end{array}\right]^{T}$. The Eigenvalues of $\mathbf{Q}^{*}$ are $\{0,0,0,-2.425,7.6195\}$ indicating an indefinite form. Therefore, the surface is crossable. A cross-section of the workspace is shown in Fig. (11a) where each curve is numbered. Non-crossable surfaces are shown in Fig. (11b) with their respective direction of admissible movement.

A trajectory planned in the workspace, e.g., the trajectory from A to B on Fig. 11a, depicts intersections with only the barrier due to surface $\Psi_{2}$. This singular surface characterizes a configuration for which $q_{2}=-\pi / 4$ and $q_{4}=q_{5}=0$ as shown in Fig. 12a. For this configuration, the singular surface is shown in Fig. 12b.

\section{CONCLUSIONS}

The problem of verifying a path trajectory in the workspace of a robot manipulator subject to joint constraints has been addressed. The manipulator may reach a singular configuration where the motion is interrupted. A general formulation for determining barriers to motion inside the workspace is presented whereby the singular behavior of the underlying mechanism is characterized in the form of parametric geometric entities. It was shown that at the intersection of a path trajectory with a barrier, the manipulator's endeffector can be analytically investigated subject to definiteness properties of a quadratic form. Admissible normal movements of the end-effector across the singular surface are determined from this quadratic form. The formulation is applicable to manipulators that can be represented using the Denavit-Hartenberg method.

It was also shown that all barriers to motion that may cause interruption to the motion are analytically determined. The intersection of a path trajectory with those surfaces can readily be determined. Therefore, although it is always possible to verify whether a 
trajectory admits an uninterrupted motion (smooth motion), it is not always possible to compute an initial configuration that does admit a smooth motion.

Although the formulation is presented using planar three-degree-of-freedom and spatial five degree-of-freedom manipulators, it is valid for the general class of serial manipulators. While the experimental computer code used to implement the numerical algorithm is not refined, it nevertheless has demonstrated a generalized method for trajectory verification of serial manipulators.

\section{REFERENCES}

1 Craig, J. J. (1989) Introduction to Robotics: Mechanics and Control, Addison Wesley Publishing.

2 Tsai, K. Y. and Kohli, D. (1993) "Trajectory Planning in Task Space for General Manipulators," Transactions of the ASME, Journal of Mechanical Design, Vol. 115, No. 4, pp. 915-921.

3 Tsai, K. Y., Kohli, D., and Arnold, J. (1993) "Trajectory Planning in Joint Space for Mechanical Manipulators," Transactions of the ASME, Journal of Mechanical Design, Vol. 115, No. 4, pp. 909-914.

4 Merlet, J.P. (1994) "Trajectory Verification in the Workspace for Parallel Manipulators," International Journal of Robotics Research, Vol. 13, No. 4, pp. 326-333.

5 Milton, C. and Jennings, D. (1994) "Path Planning for a Manipulator with Constraints on Orientation," Journal of Intelligent Robotic Systems: Theory and Applications Vol. 11, No. 1-2, pp. 67-77.

6 Althofer, K., Fraser, D.A., and Bugmann, G., (1995) "Rapid Path Planning for Robotic Manipulators Using and Emulated Resistive Grid," Electronics Letters Vol. 31, No. 22, pp. 1960-1961.

7 Seereeram, S., and Wen, J.T., (1995) "Global Approach to Path Planning for Redundant Manipulators," IEEE Transactions on Robotics and Automation, Vol. 11, No. 1, pp. 152-160.

8 Agrawal, O. P. and Xu, Y. (1994) "On the Global Optimum Path Planning for Redundant Space Manipulators," IEEE Transactions on Systems, Man and Cybernetics, Vol. 24, No. 9, pp. 1306-1316.

9 Nenchev, D.N., (1995) Tracking Manipulator Trajectories with Ordinary Singularities. A Null Space Based Approach," International Journal of Robotics Research, Vol. 14, No. 4, pp. 399-404.

10 Waldron, K. J., 1987. Operating barriers within the workspace of manipulators. Proceedings of the Society of Manufacturing Engineers, Chicago, IL, Robots II/17th ISIR, 8:35-46.

11 Nielsen, L. deWitt, C. C. and Hagander, P. 1991. Controllability issues of robots in singular configurations. Proceedings of IEEE Int. Conf. on Rob. and Autom., Sacramento, CA. 
12 Oblak, D. and Kohli, D., 1988. Boundary surfaces, limit surfaces, crossable and noncrossable surfaces in workspace of mechanical manipulators. ASME Journal of Mechanisms, Transmissions, and Automation in Design, (110):389-396.

13 Haug, E. J. Adkins, F. A. Qiu, C. C. and Yen, J. 1995. Analysis of barriers to control of manipulators within accessible output sets. Proceedings of the 20th ASME Design Engineering Technical Conference, Boston, MA, 82:697-704.

14 Abdel-Malek, K. and Yeh, H.J., (1997) "Analytical Boundary of the Workspace for General Three Degree-of-Freedom Mechanisms," International Journal of Robotics Research. Vol. 16, No. 2, pp. 198-213.

15 Abdel-Malek, K., Adkins, F., Yeh, H.J., and Haug, E.J (1997), "On the Determination of Boundaries to Manipulator Workspaces," Robotics and Computer-Integrated Manufacturing, Vol. 13, No. 1, pp.63-72.

17 Denavit, J. and Hartenberg, R.S., 1955. A kinematic notation for lower-pair mechanisms based on matrices. Journal of Applied Mechanics, 77:215-221.

18 Fu, K. S. Gonzalez, R. C. and Lee, C. S. G. 1987. Robotics: Control, Sensing, Vision, and Intelligence, McGraw-Hill, Inc., New York. 


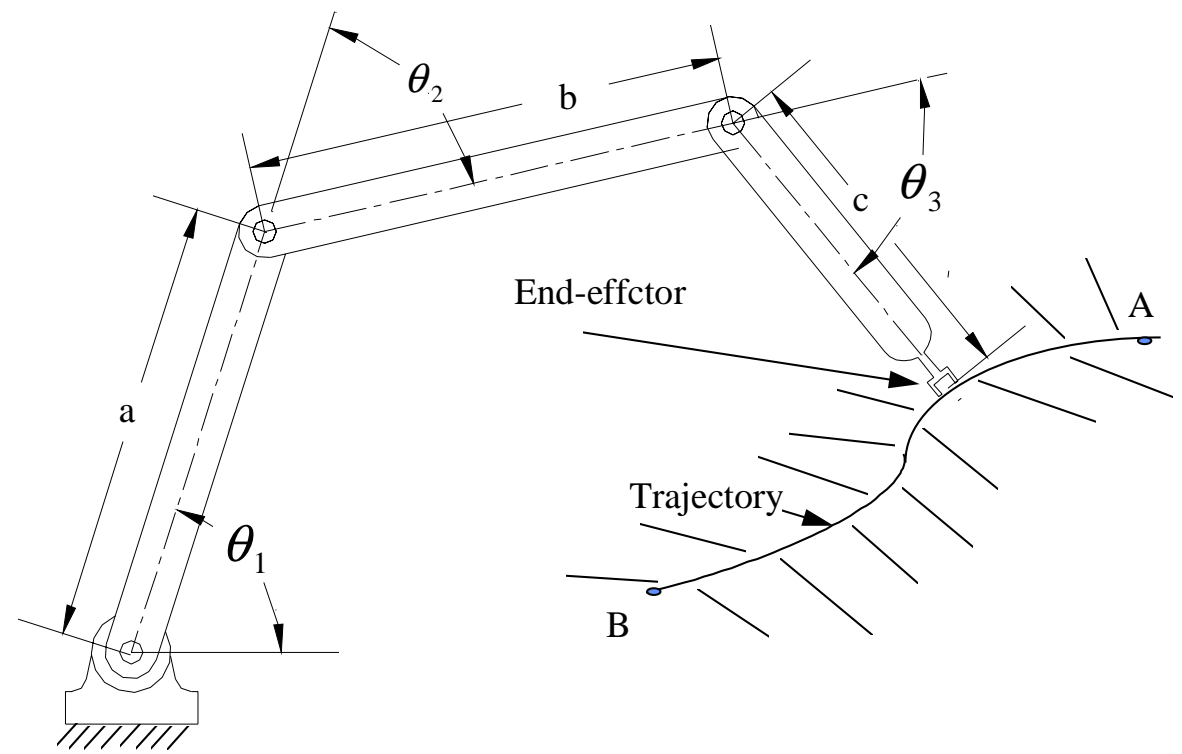

Fig. 1 A manipulator following a trajectory connecting points A and B

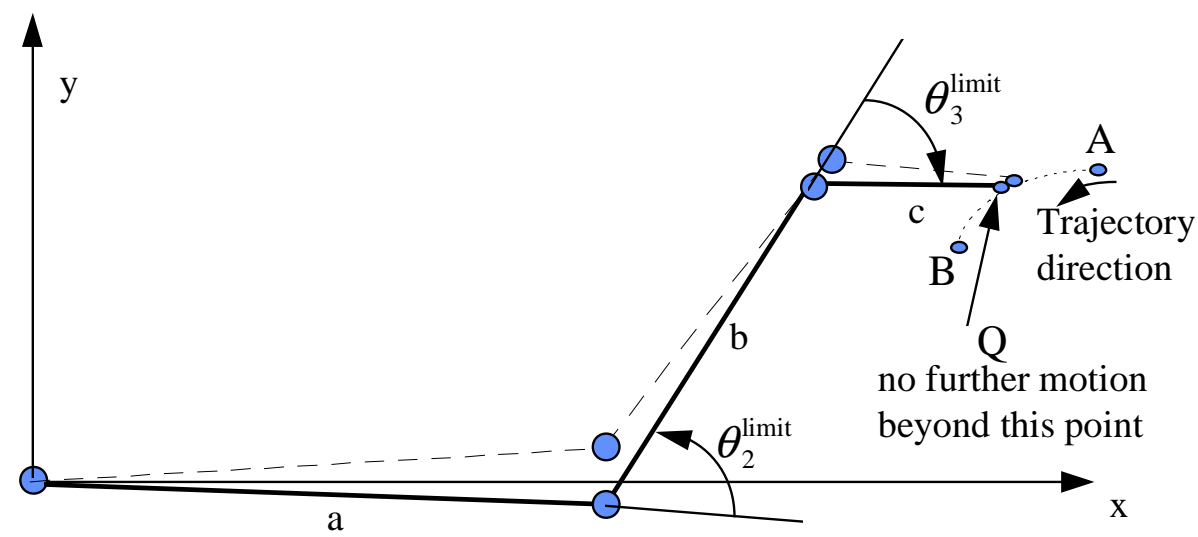

Fig. 2 A manipulator at a singular configuration interrupting the motion

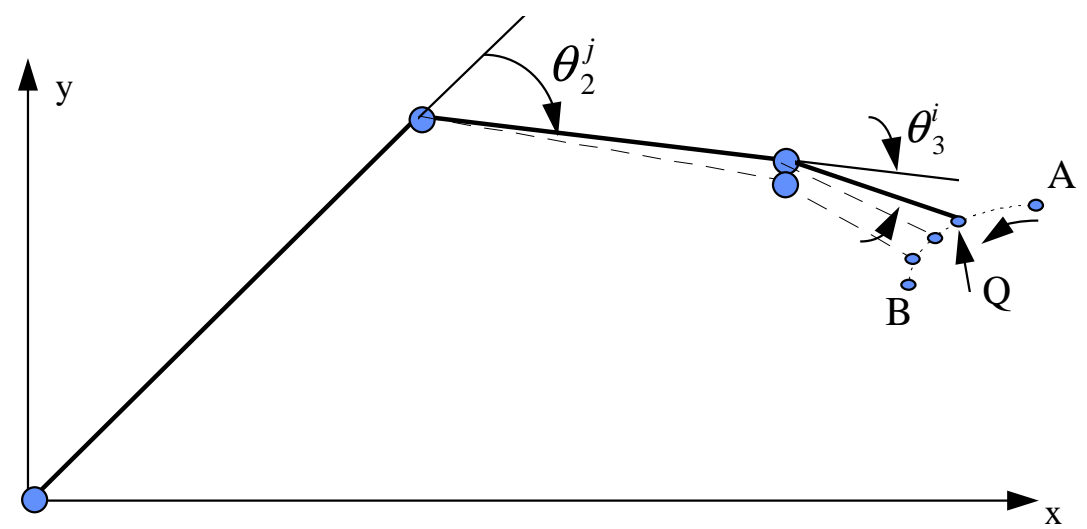

Fig. 3 A configuration that allows continuation of the motion 


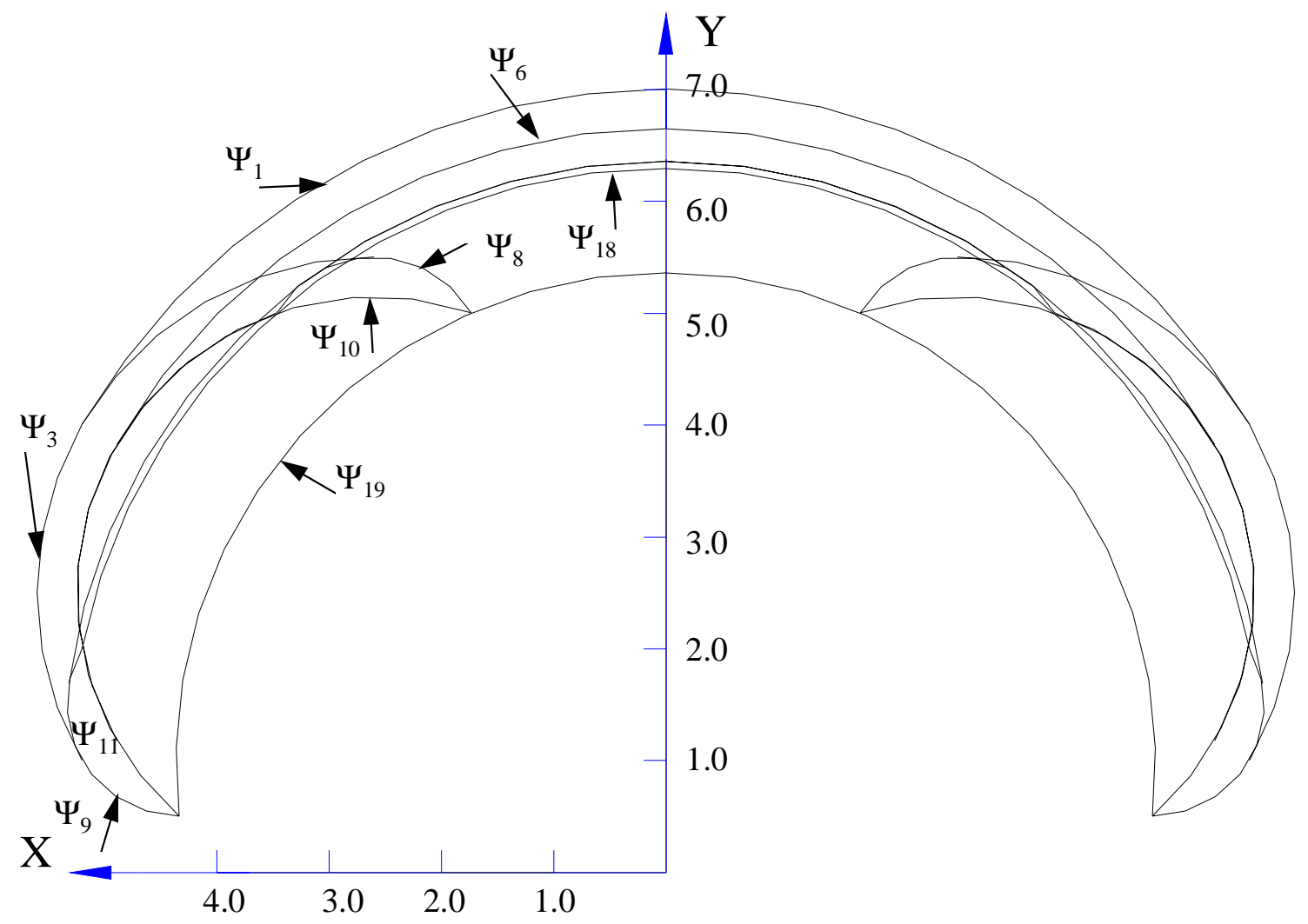

Fig. 4 Singular curves of the planar 3-bar linkage

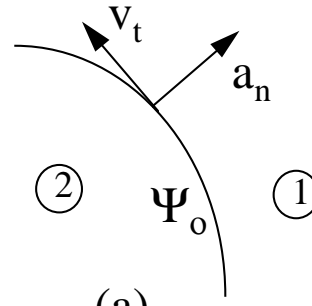

(a)

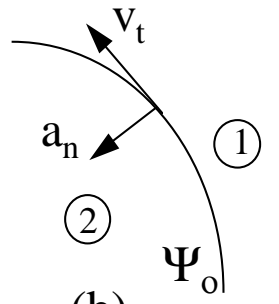

(b)

Fig. 5. (a) End-effector can move to side 1 (b) end-effector's movement into side 2 depends on $a_{n}$ and $v_{t}$ 


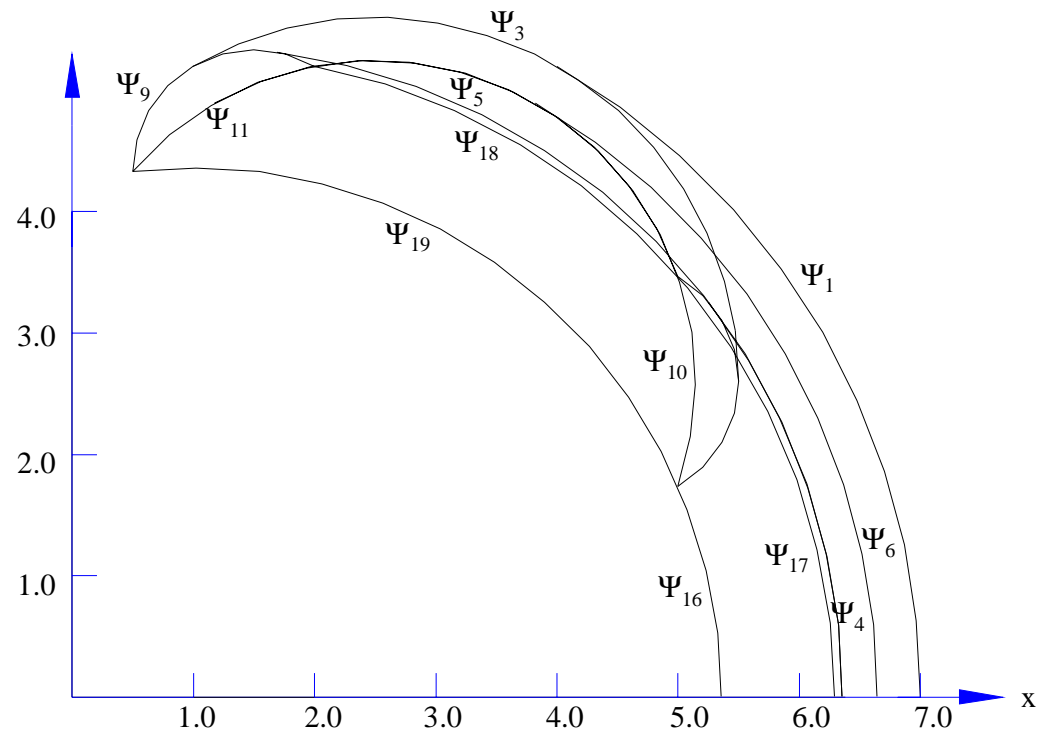

Fig. 6 (a) Singular curves

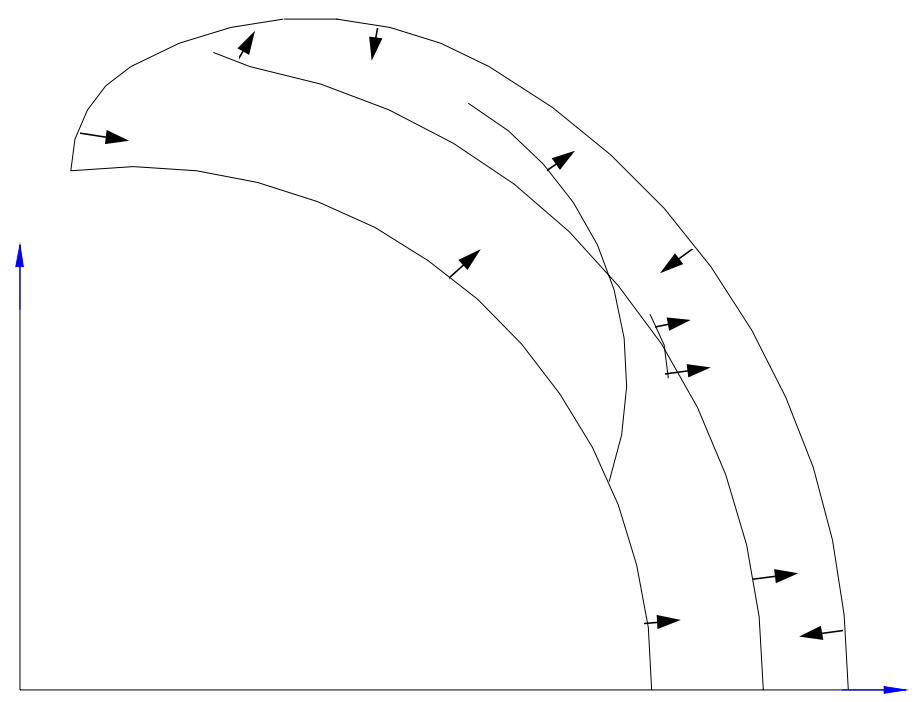

Fig. 6(b) Admissible normal movement directions 


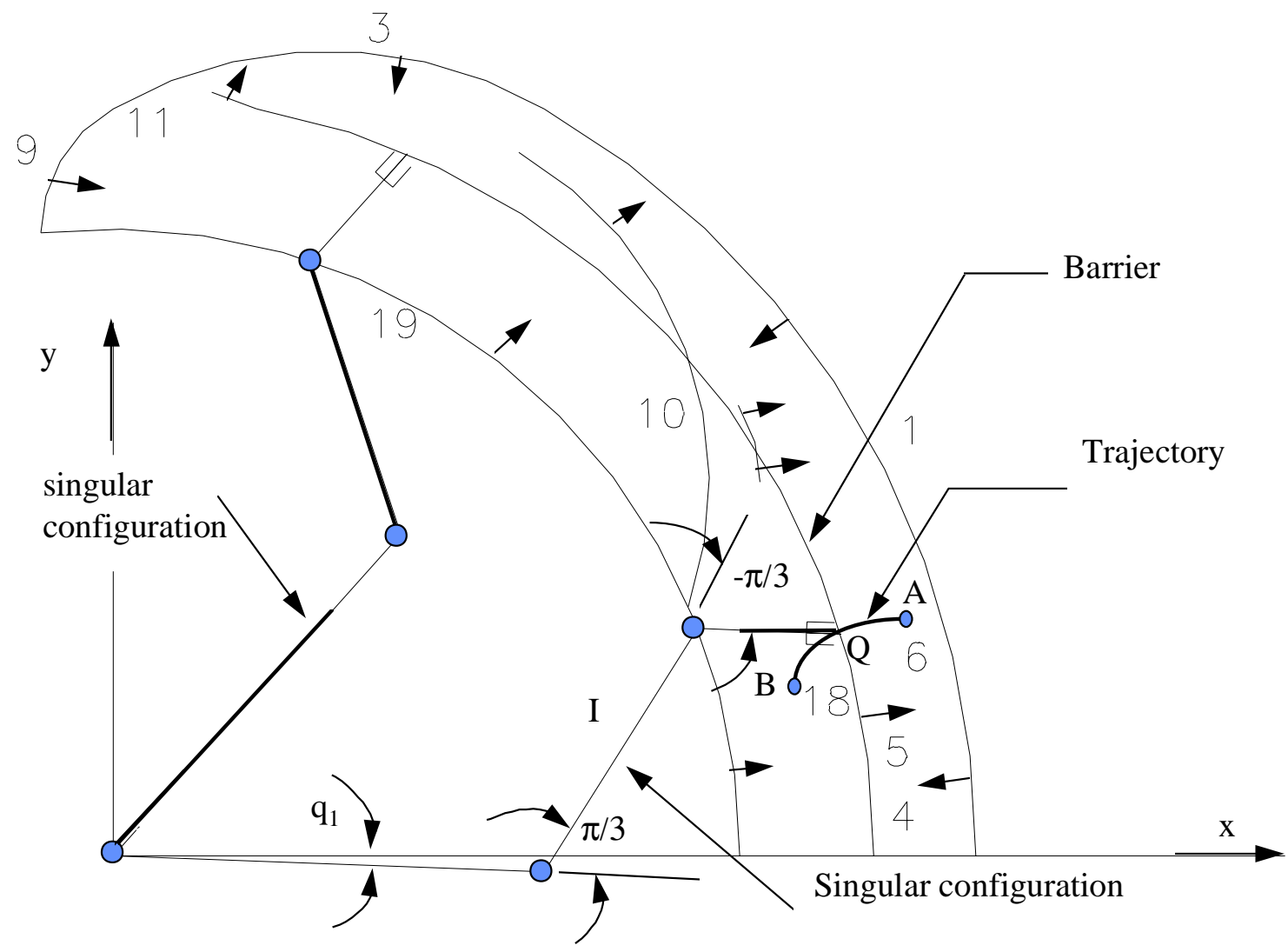

Fig. 7 The manipulator arm at a singular configuration with all non-crossable surfaces depicted

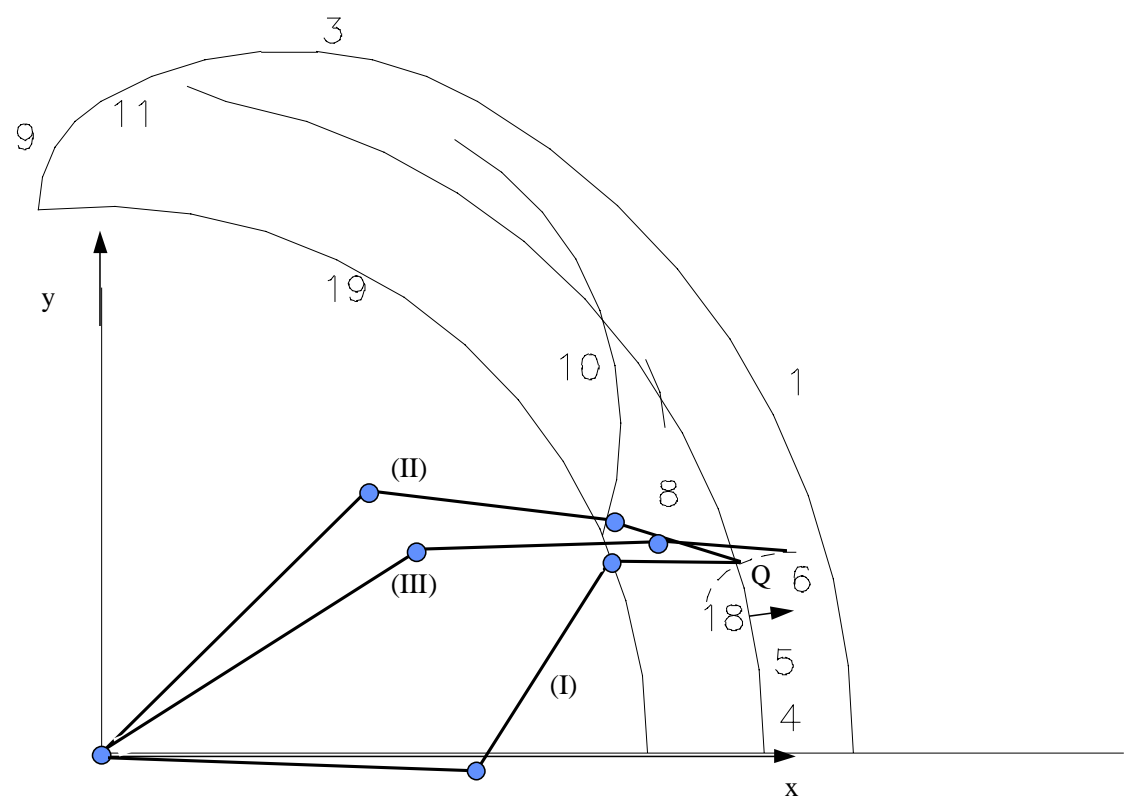

Fig. 8 The manipulator at a singular configuration (I), at an initial configuration (II), and at a configuration (III) that would allow the continuation of the motion. 


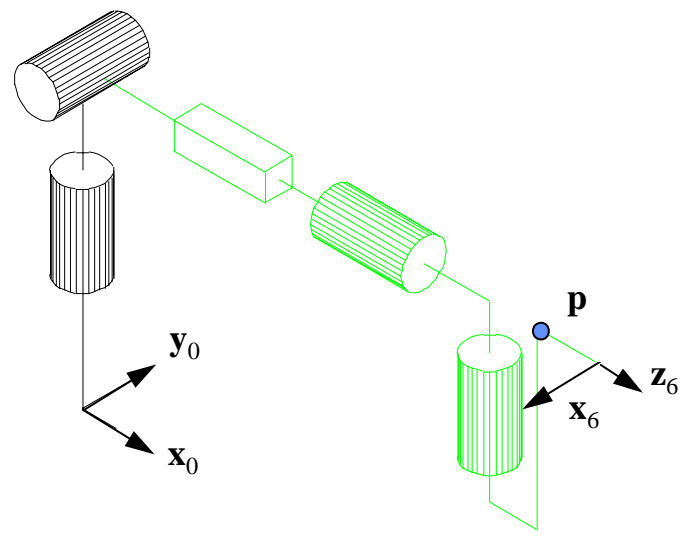

Fig. 9 A five degree-of-freedom manipulator

Fig. 10 A cross-section of the workspace of the 5-DOF manipulator 
Fig. 11 (a) Crossable surfaces and (b) Admissible directions of normal movement

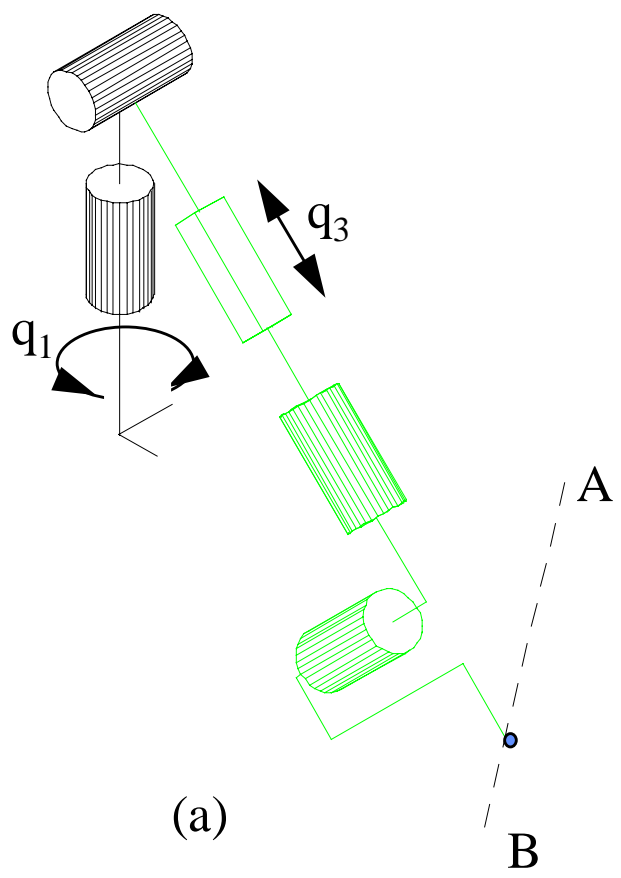

Fig. 12 (a) The manipulator in the singular configuration corresponding to $\Psi_{2}$

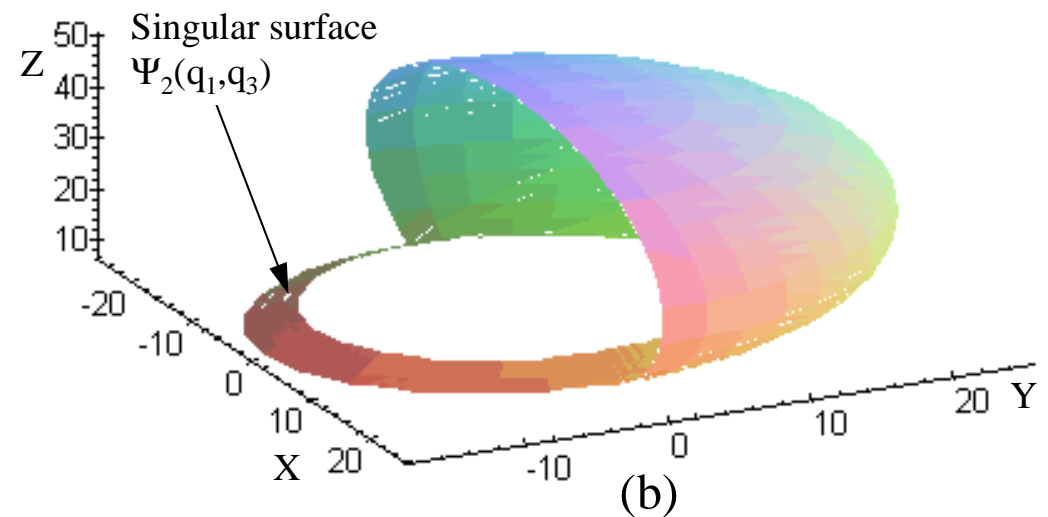

Fig. 12 (b) the singular surface $\Psi_{2}$ shown in the workspace 